\title{
Range Identification in the Presence of Unknown Motion Parameters for Perspective Vision Systems
}

\author{
Lili Ma, Chengyu Cao, Naira Hovakimyan, Warren E. Dixon, and Craig Woolsey
}

\begin{abstract}
This paper presents a nonlinear observer for the range identification problem for a moving object, whose motion is described by a known linear system with unknown coefficients. Convergent estimates of the unknown motion parameters can be guaranteed under certain observability conditions.
\end{abstract}

\section{INTRODUCTION}

A variety of 3D motion estimation algorithms have been developed since the 1970's, inspired by such disparate applications as robot navigation, medical imaging, and video conferencing. Even though motion estimation from imagery is not a new topic, continual improvements in digital imaging, computer processing capabilities, and nonlinear estimation theory have helped to keep the topic current. Existing methods for 3D motion estimation include the nonlinear optimization formulation [1]-[3], linear least-square algorithms [4], the extended Kalman filter (EKF) [5], [6], and perspective nonlinear observers [7]-[13]. Perspective nonlinear observers refer to the class of observers that arise from a control point of view in perspective dynamical systems (PDS) framework. In general, a PDS is a linear system whose output is observed up to a homogeneous line [9].

Among the aforementioned algorithms, the nonlinear optimization formulation generally suffers from the initial value selection problem, while the shortcoming of the (total) least-square algorithms, which are SVD-based, is sensitivity to noise [3]. Assuming the moving object follows certain motion dynamics, extended Kalman filter (EKF) has been used to estimate the states of a nonlinear system, where the states can be chosen to include the motion parameters and positions. The EKF is a recursive approach that usually requires less computation time for each new set of data (each new image, for example). State estimates are computed based on all past data and can readily extrapolate the state estimates ahead in time to aid in preprocessing the next set of data. The EKF is based on the linearization about an estimated trajectory. However, for the vision-based motion estimation problem, geometric structure of a perspective system will be lost if a linearization-based approach is taken. Moreover, the $\mathrm{EKF}$ is more complicated since a priori knowledge of the noise distribution is required. Due to the above mentioned problems, efforts have been made towards other nonlinear

Lili Ma, Chengyu Cao, Naira Hovakimyan, and Craig Woolsey are with Department of Aerospace and Ocean Engineering, Virginia Tech, Blacksburg, VA 24061-0203 emails: \{lma05, chengyu, nhovakim, cwoolsey\}@vt.edu

Warren E. Dixon is with Department of Mechanical and Aerospace Engineering, University of Florida, Gainesville, FL 32611-6250 emai I: wdixoneufl.edu observers for perspective dynamic systems that arise in visual tracking problems. This class of nonlinear observers is referred to as perspective nonlinear observer. This paper presents one method for such an observer design.

Perspective nonlinear observers are used quite often for determining the unknown states (i.e., the Euclidean coordinates) of a moving object with known motion parameters. For example, an identifier-based observer was proposed in [7] to estimate a stationary point's 3D position using a moving camera. Another discontinuous observer, motivated by sliding mode and adaptive methods, is developed in [9] that renders the state observation error uniformly ultimately bounded. A state estimation algorithm with a single homogeneous observation (i.e., a single image coordinate) is presented in [12]. A reduced-order nonlinear observer is described in [11] to provide asymptotic range estimates. The above described observers are based on a conventional planar imaging surface. In [14], [15], the state identification problem is discussed for a paraboloid surface. All these results are based on the assumption that the motion parameters are known. In this paper, we discuss a situation when some of the motion parameters, more specifically, the rotational parameters, are unknown constants. The objective is to achieve state estimation and parameter convergence.

One model for the relative motion of a point in the camera's field of view is the following linear system:

$$
\left[\begin{array}{c}
\dot{X}(t) \\
\dot{Y}(t) \\
\dot{Z}(t)
\end{array}\right]=\left[\begin{array}{lll}
a_{11} & a_{12} & a_{13} \\
a_{21} & a_{22} & a_{23} \\
a_{31} & a_{32} & a_{33}
\end{array}\right]\left[\begin{array}{c}
X(t) \\
Y(t) \\
Z(t)
\end{array}\right]+\left[\begin{array}{c}
b_{1} \\
b_{2} \\
b_{3}
\end{array}\right],
$$

where the matrix $\left[a_{i j}\right]$ presents the rotational dynamics, the vector $\left[b_{i}\right]$ corresponds to the translational motion, while $[X, Y, Z]^{\top}$ are the coordinates of the point in the camera frame. The affine motion dynamics introduced in (1) describes an object motion that undergoes a rotation, translation, and linear deformations [16]. From the 2D image plane, the homogeneous output observations are given by

$$
x_{1}(t)=X(t) / Z(t), \quad x_{2}(t)=Y(t) / Z(t) .
$$

These equations might model either a stationary point's 3D position as observed from a moving camera (assuming that the moving camera's velocities can be measured [7]) or a moving point's 3D position as observed from a stationary camera [16]. In general, $a_{i j}$ can be time-varying functions, but in this paper we limit the discussion to constant $a_{i j}$ 's.

Remark 1.1: Here, the $b_{j}$ 's are not constrained to be constants since, as to be seen in Assumption 3.1, the $b_{j}$ 's 
need to satisfy certain observability conditions to estimate the range information in the presence of unknown $a_{i j}$ 's.

Let

$$
\begin{aligned}
x(t) & =\left[x_{1}(t), x_{2}(t), x_{3}(t)\right]^{\top} \\
& =[X(t) / Z(t), Y(t) / Z(t), 1 / Z(t)]^{\top} .
\end{aligned}
$$

The system (1) with output observations (3) is equivalent to the system

$$
\left\{\begin{aligned}
{\left[\begin{array}{c}
\dot{x}_{1}(t) \\
\dot{x}_{2}(t)
\end{array}\right] } & =\left[\begin{array}{c}
b_{1}-b_{3} x_{1} \\
b_{2}-b_{3} x_{2}
\end{array}\right] x_{3}+\left[\begin{array}{c}
a_{13}+\left(a_{11}-a_{33}\right) x_{1} \\
a_{23}+a_{21} x_{1}
\end{array}\right] \\
& +\left[\begin{array}{c}
a_{12} x_{2}-a_{31} x_{1}^{2}-a_{32} x_{1} x_{2} \\
\left(a_{22}-a_{33}\right) x_{2}-a_{31} x_{1} x_{2}-a_{32} x_{2}^{2}
\end{array}\right], \\
\dot{x}_{3}(t) & =-\left(a_{31} x_{1}+a_{32} x_{2}+a_{33}\right) x_{3}-b_{3} x_{3}^{2},
\end{aligned}\right.
$$

with output $y(t)=\left[x_{1}(t), x_{2}(t)\right]^{\top}$.

Practically, the outputs $\left(x_{1}(t), x_{2}(t)\right)$ defined in (2) are obtained through an inverse camera imaging process when a pin-hole camera is used, as shown in Fig. 1, where the camera's focal length $f$ is assumed to be 1 without loss of generality. A $3 \mathrm{D}$ point $[X, Y, Z]^{\top}$ in the camera frame goes through perspective projection and is observed as $[X / Z, Y / Z, 1]^{\top}$. The $2 \mathrm{D}$ coordinates $[X / Z, Y / Z]^{\top}$ experience the camera's imaging process and show on the image plane as an image point with pixel-level coordinates $[u, v]^{\top} \cdot[X / Z, Y / Z]^{\top}$ can be calculated from the pixel-level coordinate assuming an ideal one-to-one mapping including camera modeling and calibration, Fig. 2. The calculated point $[X / Z, Y / Z]^{\top}$, in the camera frame, serves as input to the observer to estimate the $3 \mathrm{D}$ position $[X, Y, Z]^{\top}$.

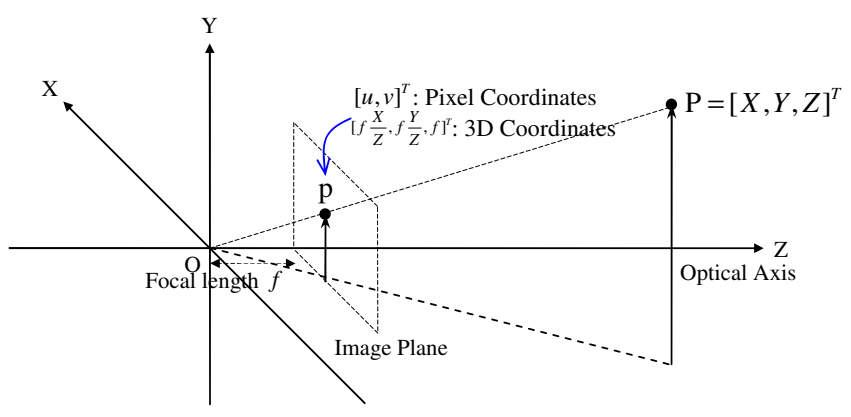

Fig. 1. Perspective projection

The range identification problem that has been discussed in the literature involves estimating $x_{3}(t)$ from measurements $\left(x_{1}(t), x_{2}(t)\right)$ assuming that the motion parameters $a_{i j}$ and $b_{i}$ in (1) are known (where $i, j \in\{1,2,3\}$ ). Here, we assume that some of the parameters $a_{i j}$ are unknown. The objective, then, is to estimate $x_{3}(t)$ as well as the unknown $a_{i j}$ 's. This problem can be formulated in a way such that an existing identifier-based observer (IBO), described in [7], can be applied. Under certain assumptions, the approach provides exponential convergence of both the range and parameter estimates. The IBO observer is motivated by adaptive filtering theory, where the state and unknown parameters of a dynamical system are estimated simultaneously. The general approach of dynamic estimation appears often in machine vision literature, including [7]-[13], to name just a few.

The paper is organized as follows. A brief review of the IBO observer is given in Sec. II. In Sec. III, the range identification problem with unknown motion parameters is presented. Section IV presents simulation results. Section V provides some summary conclusions.

\section{REVIEW OF THE IBO OBSERVER}

The IBO observer is designed for the following class of nonlinear systems:

$$
\left\{\begin{aligned}
\dot{\boldsymbol{x}}_{1} & =w^{\top}\left(\boldsymbol{x}_{1}, \boldsymbol{u}\right) \boldsymbol{x}_{2}+\phi\left(\boldsymbol{x}_{1}, \boldsymbol{u}\right), \\
\dot{\boldsymbol{x}}_{2} & =g\left(\boldsymbol{x}_{1}, \boldsymbol{x}_{2}, \boldsymbol{u}\right), \\
\boldsymbol{y} & =\boldsymbol{x}_{1},
\end{aligned}\right.
$$

where $\boldsymbol{x}_{1}(t) \in \mathbb{X}_{1} \subset \mathbb{R}^{n_{1}}, \boldsymbol{x}_{2}(t) \in \mathbb{X}_{2} \subset \mathbb{R}^{n_{2}}$ and $\boldsymbol{u}(t) \in \mathbb{U} \subset \mathbb{R}^{k}$. The $n_{1} \times n_{2}$ matrix $w^{\top}\left(\boldsymbol{x}_{1}, \boldsymbol{u}\right)$ and the vector $g\left(\boldsymbol{x}_{1}, \boldsymbol{x}_{2}, \boldsymbol{u}\right)$ are general nonlinear functions of their parameters. Let $\boldsymbol{x}=\left[\boldsymbol{x}_{1}^{\top}, \boldsymbol{x}_{2}^{\top}\right]^{\top}$ and $n=n_{1}+n_{2}$ so that $\boldsymbol{x} \in \mathbb{X} \subset \mathbb{R}^{n}$ where $\mathbb{X}=\mathbb{X}_{1} \oplus \mathbb{X}_{2}$.

Following [7], we introduce the following assumptions: Assumption 2.1:

1) Let $\boldsymbol{x}(t)$ be bounded $\|\boldsymbol{x}(t)\|<M, M>0$ for every $t \geq 0$. Let $\Omega=\left\{\boldsymbol{x} \in \mathbb{R}^{n}:\|\boldsymbol{x}(t)\|<M\right\}$. Further, for some fixed constant $\gamma>1$, let $\Omega_{\gamma}=\{\boldsymbol{x} \in$ $\left.\mathbb{R}^{n}:\|\boldsymbol{x}(t)\|<\gamma M\right\}$. Assume further that the function $g\left(\boldsymbol{x}_{1}, \boldsymbol{x}_{2}, \boldsymbol{u}\right)$ is locally Lipschitz in $\Omega_{\gamma}$ with respect to $\boldsymbol{x}_{2}$, i.e., there exists a positive constant $\alpha$ such that

$$
\left\|g\left(\boldsymbol{x}_{1}, \boldsymbol{x}_{2}, \boldsymbol{u}\right)-g\left(\boldsymbol{x}_{1}, \boldsymbol{z}_{2}, \boldsymbol{u}\right)\right\|<\alpha\left\|\boldsymbol{x}_{2}-\boldsymbol{z}_{2}\right\|,
$$

for all $\boldsymbol{x}_{2}, \boldsymbol{z}_{2} \in \Omega_{\gamma} \cap \mathbb{X}_{2}$, uniformly in $\boldsymbol{x}_{1} \in \Omega_{\gamma} \cap \mathbb{X}_{1}$ and $\boldsymbol{u} \in \mathbb{U}$.

2) Let the regressor matrix $w^{\top}\left(\boldsymbol{x}_{1}, \boldsymbol{u}\right)$ and its first time derivative be piecewise smooth and uniformly bounded. Further, assume that there exist positive constants $L_{1}, L_{2}, \eta$ and $\mu$ such that

$$
\begin{gathered}
\left\|w^{\top}\left(\boldsymbol{x}_{1}, \boldsymbol{u}\right)\right\|<L_{1}, \quad\left\|\frac{\mathrm{d} w^{\top}\left(\boldsymbol{x}_{1}, \boldsymbol{u}\right)}{\mathrm{d} t}\right\|<L_{2}, \\
\int_{t}^{t+\mu} w\left(\boldsymbol{x}_{1}(\tau), \boldsymbol{u}(\tau)\right) w^{\top}\left(\boldsymbol{x}_{1}(\tau), \boldsymbol{u}(\tau)\right) \mathrm{d} \tau>\eta I,
\end{gathered}
$$

for all $t \geq 0$, for all trajectories that originate in $\mathbb{X}$ and for all $\boldsymbol{u} \in \mathbb{U}$, while $I$ denotes the identity matrix of an appropriate dimension.

Letting $\hat{\boldsymbol{x}}_{1}$ and $\hat{\boldsymbol{x}}_{2}$ be the estimates of $\boldsymbol{x}_{1}$ and $\boldsymbol{x}_{2}$, respectively, the IBO is introduced as:

$$
\left\{\begin{array}{l}
\dot{\hat{\boldsymbol{x}}}_{1}=G A\left(\hat{\boldsymbol{x}}_{1}-\boldsymbol{x}_{1}\right)+w^{\top}\left(\boldsymbol{x}_{1}, \boldsymbol{u}\right) \hat{\boldsymbol{x}}_{2}+\phi\left(\boldsymbol{x}_{1}, \boldsymbol{u}\right), \\
\dot{\hat{\boldsymbol{x}}}_{2}=-G^{2} w\left(\boldsymbol{x}_{1}, \boldsymbol{u}\right) P\left(\hat{\boldsymbol{x}}_{1}-\boldsymbol{x}_{1}\right)+g\left(\boldsymbol{x}_{1}, \hat{\boldsymbol{x}}_{2}, \boldsymbol{u}\right), \\
\hat{\boldsymbol{x}}\left(t_{i}^{+}\right)=M \frac{\hat{\boldsymbol{x}}\left(t_{i}^{-}\right)}{\left\|\hat{\boldsymbol{x}}\left(t_{i}^{-}\right)\right\|},
\end{array}\right.
$$

where $G$ is a constant and $A$ is an $n_{1} \times n_{1}$ Hurwitz matrix. The matrix $P$ is the positive definite solution of the Lyapunov equation $A^{\top} P+P A=-Q$ where $Q$ is a positive-definite symmetric matrix, and the sequence $t_{i}$ is defined as

$$
t_{i}=\min \left\{t: t>t_{i-1} \text { and }\|\hat{\boldsymbol{x}}(t)\| \geq \gamma M\right\}, t_{0}=0,
$$




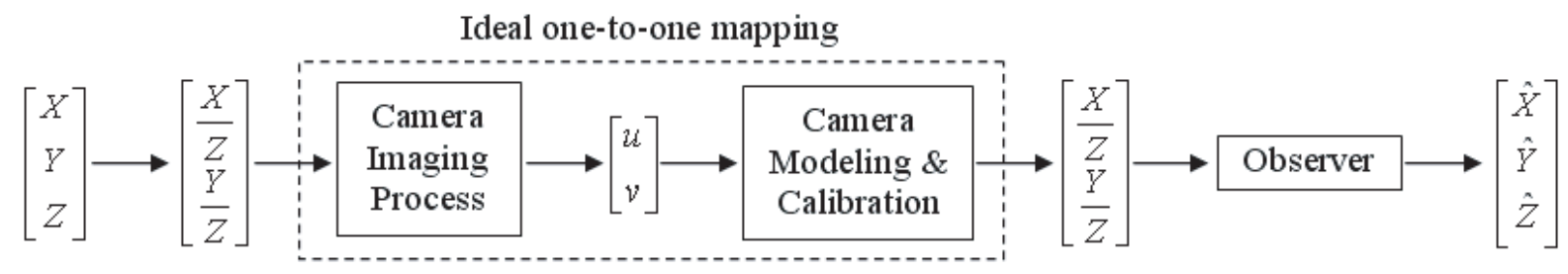

Fig. 2. Range identification with camera modeling

where $\gamma$ is a fixed constant.

Remark 2.1: $t_{i}$ defined in (9) are the discontinuity points of the system (8). It follows from (8) and (9) that $\hat{\boldsymbol{x}}(t)$ is bounded: $\|\hat{\boldsymbol{x}}(t)\| \leq \gamma M$. The state of the original nonlinear system is assumed to be bounded as stated in Assumption 2.1 .

Remark 2.2: The IBO observer in (8) is a discontinuous observer due to its third equation. Because the observer has discontinuous right-hand side, its solution is interpreted in Filippov's sense [17]. A continuous observer can be obtained by applying the projection operator [18]:

$$
\begin{aligned}
& \dot{\hat{\boldsymbol{x}}}(t)= \\
& \operatorname{Proj}\left(\left[\begin{array}{l}
G A\left(\hat{\boldsymbol{x}}_{1}-\boldsymbol{x}_{1}\right)+w^{\top}\left(\boldsymbol{x}_{1}, \boldsymbol{u}\right) \hat{\boldsymbol{x}}_{2}+\phi\left(\boldsymbol{x}_{1}, \boldsymbol{u}\right) \\
-G^{2} w\left(\boldsymbol{x}_{1}, \boldsymbol{u}\right) P\left(\hat{\boldsymbol{x}}_{1}-\boldsymbol{x}_{1}\right)+g\left(\boldsymbol{x}_{1}, \hat{\boldsymbol{x}}_{2}, \boldsymbol{u}\right)
\end{array}\right], \hat{\boldsymbol{x}}(t)\right) .
\end{aligned}
$$

Theorem 2.1: [7] Subject to Assumption 2.1, there exists a positive constant $G_{0}$ such that the estimation errors $\boldsymbol{e}_{1}=$ $\boldsymbol{x}_{1}-\hat{\boldsymbol{x}}_{1}$ and $\boldsymbol{e}_{2}=\boldsymbol{x}_{2}-\hat{\boldsymbol{x}}_{2}$ converge to zero exponentially if the constant $G$ in (8) is chosen larger than $G_{0}$.

In Ref. [7], the IBO has been applied to the range identification problem assuming that all the motion parameters in (1) are known. In this paper, some of the $a_{i j}$ 's in (1) are assumed to be unknown constants. Under this assumption, the unknown parameters can be treated as additional states of a nonlinear system to which the IBO can be applied. This is given in Sec. III.

\section{Range IdENTIFICATION With UnKNOWn Motion PARAMETERS}

In this section, we consider the state estimation problem for the perspective dynamic system (4), where the motion parameters $a_{i j}$ for $i, j=1,2,3$ (except for $a_{33}$ ) are assumed to be unknown constants. Here, $a_{33}$ is assumed to be known to satisfy the observability condition for estimating both the state and the unknown motion parameters. This will be elaborated in Remark 3.3.

Let $\boldsymbol{\theta}$ be a vector of these unknown constants defined as

$$
\boldsymbol{\theta}=\left[a_{11}, a_{12}, a_{13}, a_{21}, a_{22}, a_{23}, a_{31}, a_{32}\right]^{\top} .
$$

The system (4) can be rewritten as

$$
\begin{aligned}
& {\left[\begin{array}{c}
\dot{x}_{1}(t) \\
\dot{x}_{2}(t)
\end{array}\right]=w_{s}^{\top}\left(x_{1}, x_{2}\right)\left[\begin{array}{c}
x_{3} \\
\boldsymbol{\theta}
\end{array}\right]-a_{33}\left[\begin{array}{l}
x_{1} \\
x_{2}
\end{array}\right]} \\
& {\left[\begin{array}{c}
\dot{x}_{3}(t) \\
\dot{\boldsymbol{\theta}}
\end{array}\right]=\left[\begin{array}{c}
-\left(a_{31} x_{1}+a_{32} x_{2}+a_{33}\right) x_{3}-b_{3} x_{3}^{2} \\
g_{s}\left(x_{1}, x_{2}, x_{3}, a_{3 j}\right) \\
0
\end{array}\right]}
\end{aligned}
$$

with

$$
w_{s}^{\top}\left(x_{1}, x_{2}\right)=\left[\begin{array}{ccccccc}
b_{1}-b_{3} x_{1} & x_{1} & x_{2} & 1 & 0 & 0 & 0 \\
b_{2}-b_{3} x_{2} & 0 & 0 & 0 & x_{1} & x_{2} & 1 \\
& & -x_{1}^{2} & -x_{1} x_{2} \\
& -x_{1} x_{2} & & -x_{2}^{2}
\end{array}\right],
$$

which fits into the form of the general nonlinear system (5) to which IBO might be applicable, by regarding $\boldsymbol{x}_{1}=$ $\left[x_{1}, x_{2}\right]^{\top}, \boldsymbol{x}_{2}=\left[x_{3}, \boldsymbol{\theta}^{\top}\right]^{\top}$, and $\phi\left(x_{1}, \boldsymbol{u}\right)=0$. To apply the IBO observer, we need the following assumption for the system in (12).

Assumption 3.1:

1) The motion parameters $a_{i j}$ and $b_{i}(t) \forall i, j=1,2,3$ introduced in (1) are bounded functions of time.

2) Let $\boldsymbol{x}(t)=\left[x_{1}(t), x_{2}(t), x_{3}(t), \boldsymbol{\theta}^{\top}\right]^{\top}$ be bounded $\|\boldsymbol{x}(t)\|<M, M>0$ for every $t \geq 0$. Let $\Omega=$ $\left\{\boldsymbol{x} \in \mathbb{R}^{11}:\|\boldsymbol{x}(t)\|<M\right\}$. Further, for some fixed constant $\gamma>1$, let $\Omega_{\gamma}=\left\{\boldsymbol{x} \in \mathbb{R}^{11}:\|\boldsymbol{x}(t)\|<\gamma M\right\}$.

3) There do not exist constants $\kappa_{i}$ (for $i=1,2,3,4$ ) with $\sum_{i=1}^{4} \kappa_{i}^{2} \neq 0$ such that

$$
\kappa_{1} x_{1}(\tau)+\kappa_{2} x_{2}(\tau)+\kappa_{3}+\kappa_{4} b_{3}(\tau)=0,
$$

for all $\tau \in[t, t+\mu]$, where $\mu$ is a very small positive constant.

Remark 3.1: The first two statements in Assumption 3.1 are standard assumptions (see also [7], [8], [10]) that are practically properties of the physical system rather than assumptions.

Remark 3.2: The mathematical meaning of the observability condition in (14) is that all columns in $w_{s}\left(x_{1}, x_{2}\right)$ need to be linearly independent at any time interval $\tau \in$ $[t, t+\mu]$. The physical meaning is that the motion dynamics should be sufficiently exciting for the state and the motion parameters to be observable.

Remark 3.3: Assuming that the $\left[a_{i j}\right]$ (for $i, j=1,2,3$ ) are unknown constants, estimation of the state, along with the unknown parameters, cannot be achieved. This is elaborated in the next. Suppose that the $\left[a_{i j}\right]$ (for $i, j=1,2,3)$ are unknown constants and let $\theta_{9 \times 1}$ be a vector of these unknown constants as $\theta_{9 \times 1}=$ $\left[a_{11}, a_{12}, a_{13}, a_{21}, a_{22}, a_{23}, a_{31}, a_{32}, a_{33}\right]^{\top}$. The system (12a) can be written as

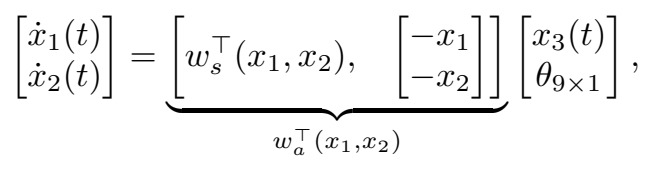


where $w_{s}^{\top}\left(x_{1}, x_{2}\right)$ is given in (13). It is obvious that the column vectors in $w_{a}^{\top}\left(x_{1}, x_{2}\right)$ in (15) are linearly dependent. More exactly, column 10 can be written as column $10=$ - column $_{6}-$ column $_{2}$. The observability condition in (7b) is thus violated.

It is straightforward to verify that the system in (12) satisfies Assumption 2.1. Indeed, for all $\boldsymbol{x}_{1} \in \Omega_{\gamma} \cap \mathbb{X}_{1}$ and $\boldsymbol{x}_{2}, \overline{\boldsymbol{x}}_{2} \in \Omega_{\gamma} \cap \mathbb{X}_{2}$, we have:

$$
\begin{aligned}
& \left\|g\left(\boldsymbol{x}_{1}, \overline{\boldsymbol{x}}_{2}\right)-g\left(\boldsymbol{x}_{1}, \boldsymbol{x}_{2}\right)\right\| \\
= & \left\|\left[g_{s}\left(x_{1}, x_{2}, \bar{x}_{3}, \bar{a}_{31}, \bar{a}_{32}, a_{33}\right)-g_{s}\left(x_{1}, x_{2}, x_{3}, a_{3 j}\right)\right]\right\| \\
0 & \|\left[\begin{array}{c}
0_{8 \times 1} \\
=
\end{array}\right. \\
= & \left\|g_{s}\left(x_{1}, x_{2}, \bar{x}_{3}, \bar{a}_{31}, \bar{a}_{32}, a_{33}\right)-g_{s}\left(x_{1}, x_{2}, x_{3}, a_{3 j}\right)\right\| \\
= & \|\left(\bar{a}_{31} x_{1}+\bar{a}_{32} x_{2}+a_{33}\right) \bar{x}_{3}+b_{3} \bar{x}_{3}^{2} \\
& -\left(a_{31} x_{1}+a_{32} x_{2}+a_{33}\right) x_{3}-b_{3} x_{3}^{2} \| \\
\leq \quad & \left(\left\|\bar{a}_{31}\right\|\left\|x_{1}\right\|+\left\|\bar{a}_{32}\right\|\left\|x_{2}\right\|+\left\|a_{33}\right\|\right)\left\|\bar{x}_{3}-x_{3}\right\| \\
& +\left\|b_{3}\right\|\left\|\bar{x}_{3}+x_{3}\right\|\left\|\bar{x}_{3}-x_{3}\right\|+\left\|x_{3}\right\|\left(\left\|x_{1}\right\|\left\|\bar{a}_{31}-a_{31}\right\|\right. \\
& \left.+\left\|x_{2}\right\|\left\|\bar{a}_{32}-a_{32}\right\|\right) \\
\leq \quad & {\left[\left\|\bar{a}_{31}\right\|\left\|x_{1}\right\|+\left\|\bar{a}_{32}\right\|\left\|x_{2}\right\|+\left\|a_{33}\right\|+\left\|b_{3}\right\|\left\|\bar{x}_{3}+x_{3}\right\|\right.} \\
& \left.+\left\|x_{3}\right\|\left(\left\|x_{1}\right\|+\left\|x_{2}\right\|\right)\right] \cdot\left\|\left[\bar{x}_{3}, \bar{a}_{31}, \bar{a}_{32}\right]^{\top}-\left[x_{3}, a_{31}, a_{32}\right]^{\top}\right\| \\
\leq \quad & \left(4 M^{2}+M_{a}+2 M M_{b}\right)\left\|\overline{\boldsymbol{x}}_{2}-\boldsymbol{x}_{2}\right\|,
\end{aligned}
$$

where it is assumed that $a_{33}$ and $b_{3}(t)$ are bounded by $M_{a}$ and $M_{b}$, respectively. That is, $\left\|a_{33}\right\| \leq M_{a}$ and $\left\|b_{3}(t)\right\| \leq$ $M_{b}, \forall t \geq 0$ (Assumption 3.1). Hence the Lipschitz condition (6) is satisfied with a Lipschitz constant $4 M^{2}+M_{a}+2 M M_{b}$.

It is also straightforward to verify that $w_{s}\left(x_{1}, x_{2}\right)$ and its first time derivative are piecewise smooth and uniformly bounded. The persistency of excitation observability condition in (7b) can be proved using the condition in (14). Let $w_{i}(t)$ be the $i^{\text {th }}$ column of $w_{s}^{\top}$ in (12) (for $i=1, \ldots, 9$ ). From Assumption 3.1, there do not exist constants $k_{i}$ (for $i=1, \ldots, 9)$ with $\sum_{i=1}^{9} k_{i}^{2} \neq 0$ such that $\sum_{i=1}^{9} k_{i} w_{i}(t)=$ 0 . Therefore, for any nonzero $9 \times 1$ vector $v$ with $\|v\|=1$, we have $v^{\top} w_{s} w_{s}^{\top} v>\varepsilon\|v\|^{2}=\varepsilon$. Thus $w_{s} w_{s}^{\top}>\varepsilon I$ and (7b) is satisfied. Since Assumption 3.1 leads to Assumption 2.1, state estimation of $x_{3}(t)$, together with the unknown motion parameters $\boldsymbol{\theta}$, can be obtained via direct application of the IBO observer.

Letting $e_{1}=\hat{x}_{1}-x_{1}, e_{2}=\hat{x}_{2}-x_{2}, e_{3}=\hat{x}_{3}-x_{3}$, the following observer can be designed for the system in (12)

$$
\left\{\begin{array}{l}
{\left[\begin{array}{c}
\dot{\hat{x}}_{1} \\
\hat{\hat{x}}_{2}
\end{array}\right]=G A\left[\begin{array}{l}
e_{1} \\
e_{2}
\end{array}\right]+w_{s}^{\top}\left(x_{1}, x_{2}\right)\left[\begin{array}{c}
\hat{x}_{3} \\
\hat{\boldsymbol{\theta}}
\end{array}\right]-a_{33}\left[\begin{array}{l}
x_{1} \\
x_{2}
\end{array}\right],} \\
{\left[\begin{array}{c}
\dot{\hat{x}}_{3} \\
\dot{\hat{\boldsymbol{\theta}}}
\end{array}\right]=-G^{2} w_{s}\left(x_{1}, x_{2}\right) P\left[\begin{array}{l}
e_{1} \\
e_{2}
\end{array}\right]+\left[\begin{array}{c}
g_{s}\left(x_{1}, x_{2}, \hat{x}_{3}, \hat{a}_{31}, \hat{a}_{32}, a_{33}\right) \\
0_{8 \times 1}
\end{array}\right],} \\
\hat{\boldsymbol{x}}\left(t_{i}^{+}\right)=M \frac{\hat{\boldsymbol{x}}\left(t_{i}^{-}\right)}{\left\|\hat{\boldsymbol{x}}\left(t_{i}^{-}\right)\right\|},
\end{array}\right.
$$

where $\boldsymbol{x}$ denotes $\left[x_{1}, x_{2}, x_{3}, \boldsymbol{\theta}^{\top}\right]^{\top}, \hat{\boldsymbol{\theta}}$ denotes the estimation of $\boldsymbol{\theta}$, and the sequence of $t_{i}$ is defined as in (9). The closedloop error dynamics can be derived from (12) and (16) as

$$
\left\{\begin{aligned}
{\left[\begin{array}{c}
\dot{e}_{1} \\
\dot{e}_{2}
\end{array}\right]=} & G A\left[\begin{array}{l}
e_{1} \\
e_{2}
\end{array}\right]+w_{s}^{\top}\left(x_{1}, x_{2}\right)\left[\begin{array}{c}
e_{3} \\
\tilde{\boldsymbol{\theta}}
\end{array}\right] \\
{\left[\begin{array}{c}
\dot{e}_{3} \\
\dot{\boldsymbol{\theta}}
\end{array}\right]=} & -G^{2} w_{s}\left(x_{1}, x_{2}\right) P\left[\begin{array}{c}
e_{1} \\
e_{2}
\end{array}\right] \\
& +\left[\begin{array}{c}
g_{s}\left(x_{1}, x_{2}, \hat{x}_{3}, \hat{a}_{31}, \hat{a}_{32}, a_{33}\right)-g_{s}\left(x_{1}, x_{2}, x_{3}, a_{3 j}\right) \\
0_{8 \times 1}
\end{array}\right]
\end{aligned}\right.
$$

where $\tilde{\boldsymbol{\theta}}=\hat{\boldsymbol{\theta}}-\boldsymbol{\theta}$ and $\dot{\tilde{\boldsymbol{\theta}}}=\dot{\hat{\boldsymbol{\theta}}}$ since $\boldsymbol{\theta}$ is assumed to be a constant vector.

According to Theorem 2.1, there exists a positive constant $G_{0}$, such that the estimation errors $\left[e_{1}, e_{2}, e_{3}, \tilde{\boldsymbol{\theta}}^{\top}\right]^{\top}$ converge to zero exponentially if the constant $G$ in (16) is chosen to be larger than $G_{0}$. Proof of IBO estimation error convergence is provided in [7].

\section{Simulation Results}

State estimation of $\left[x_{3}(t), \boldsymbol{\theta}^{\top}\right]^{\top}$ using the nonlinear observer (16) is implemented in Matlab, where the motion dynamics are selected to be

$$
\begin{aligned}
& {\left[\begin{array}{l}
\dot{X}(t) \\
\dot{Y}(t) \\
\dot{Z}(t)
\end{array}\right]=\left[\begin{array}{ccc}
0.1 & -4 & 0.5 \\
4 & -0.1 & 0.4 \\
-0.5 & -0.4 & 0
\end{array}\right]\left[\begin{array}{c}
X(t) \\
Y(t) \\
Z(t)
\end{array}\right]+\left[\begin{array}{c}
0 \\
2 \pi \sin (2 \pi t) \\
2 \pi \cos (2 \pi t)
\end{array}\right],} \\
& \left(X_{0}, Y_{0}, Z_{0}\right)=(1,1.5,2.5), \quad x_{0}=\left(X_{0} / Z_{0}, Y_{0} / Z_{0}, 1 / Z_{0}\right) .
\end{aligned}
$$

In all the simulations, the output is corrupted with uniform noise bounded by $\pm 10^{-2}$. The observer's initial states $\left(\hat{x}_{1}(0), \hat{x}_{2}(0), \hat{x}_{3}(0)\right)$ are chosen to be $\left(x_{1}(0), x_{2}(0), 1\right)$ and $\hat{\boldsymbol{\theta}}(0)=0$. The observer parameters are chosen to be $M=$ $10, A=I_{2}, P=-1 / 2 \times I_{2}, G=20$, where $I_{2}$ denotes the $2 \times 2$ identity matrix. State estimation of $x_{3}$ and parameter estimations of $a_{i j}$ for $i, j=1,2,3$ (except for $a_{33}$ ) are shown in Figs. 3 6, respectively. It can be seen that good estimates can be obtained. The $3 \mathrm{D}$ and $2 \mathrm{D}$ trajectories of the moving point and their estimates are shown in Figs. 7 and 8, for $t \in[20,25]$.

Figures $9 \sim 12$ show simulation results of the following motion dynamics

$$
\begin{gathered}
{\left[\begin{array}{c}
\dot{X}(t) \\
\dot{Y}(t) \\
\dot{Z}(t)
\end{array}\right]=\left[\begin{array}{ccc}
0.3 & -4 & 0.3 \\
4 & -0.2 & 0.4 \\
-0.6 & -0.5 & 0.05
\end{array}\right]\left[\begin{array}{c}
X(t) \\
Y(t) \\
Z(t)
\end{array}\right]+\left[\begin{array}{c}
0 \\
2 \pi \sin (3 \pi t) \\
2 \pi \cos (3 \pi t)
\end{array}\right],} \\
\left(X_{0}, Y_{0}, Z_{0}\right)=(1,1.5,2.5), x_{0}=\left(X_{0} / Z_{0}, Y_{0} / Z_{0}, 1 / Z_{0}\right),
\end{gathered}
$$

using the same design. It can be observed that both state estimation of $x_{3}(t)$ and parameter estimation of the unknown $a_{i j}$ 's are obtained.

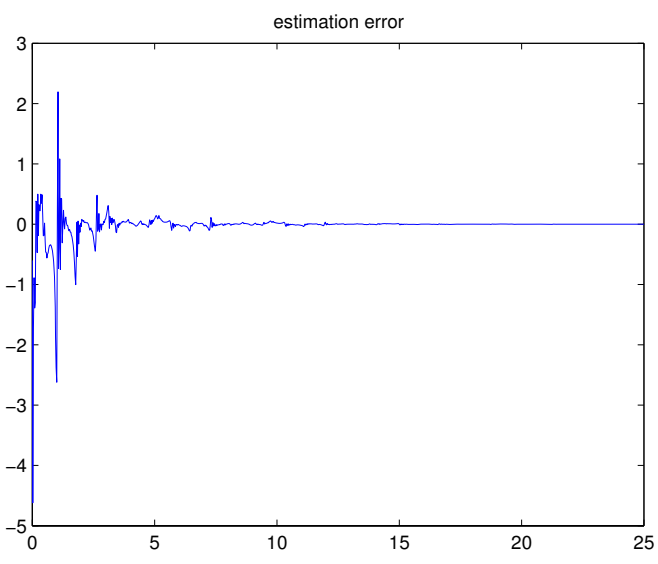

Fig. 3. State estimation error of $x_{3}$ of (18) 

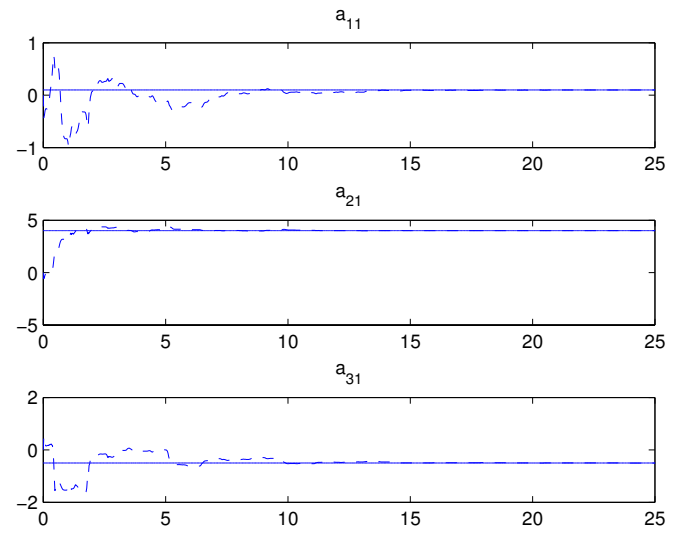

Fig. 4. Parameter estimation of $a_{i 1}$ (for $i=1,2,3$ ) of (18)
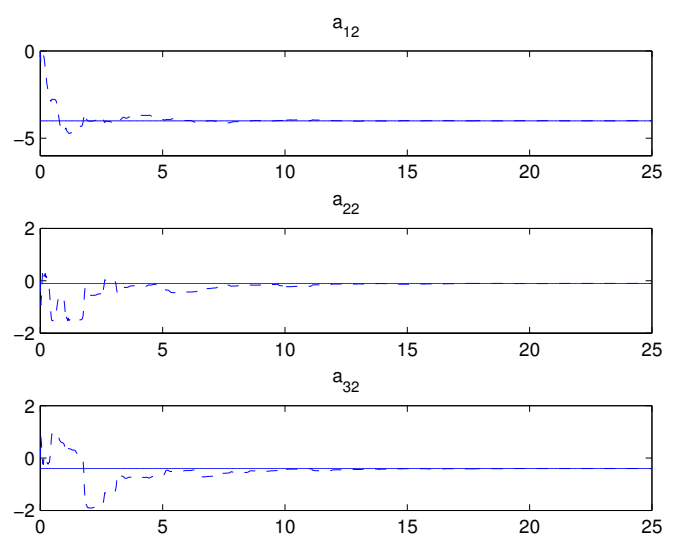

Fig. 5. Parameter estimation of $a_{i 2}$ (for $i=1,2,3$ ) of (18)

\section{CONCLUSION}

A nonlinear observer is designed to estimate the 3D positions and unknown motion parameters of a moving feature point with partially-known motion dynamics. Specifically, an affine motion with unknown constant rotational parameters is considered. The objective is to achieve state estimation (the unknown depth in the vision problem) along with parameter convergence. An existing nonlinear observer, the identifier-based observer, is utilized to achieve the objective. Convergent estimates of the state and the unknown motion parameters can be guaranteed under certain observability conditions. The proposed method is motivated by adaptive control techniques.

\section{ACKNOWLEDGMENTS}

This work was sponsored in part by ONR Grant \#N0001406-1-0801 and AFOSR MURI subcontract F49620-03-10401.

\section{REFERENCES}

[1] H. R. Cho, K. M. Lee, and S. U. Lee, "A new robust 3D motion estimation under perspective projection," in IEEE International Con-
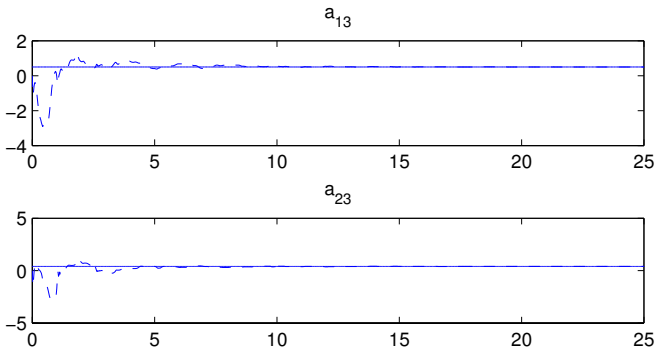

Fig. 6. Parameter estimation of $a_{13}$ and $a_{23}$ of (18)

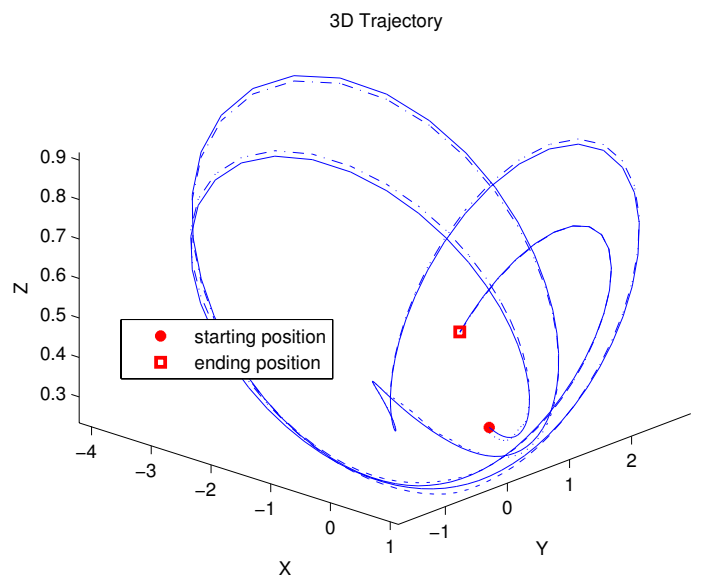

Fig. 7. 3D trajectory in the camera-centered 3D space of (18)

ference on Image Processing, Thessaloniki, Greece, Oct. 7-10 2001, pp. 660-663.

[2] K. I. Diamantaras, T. Papadimitrion, M. G. Strintzis, and M. Roumeliotis, "Total least squares 3-D motion estimation," in IEEE International Conference on Image Processing, Oct. 1998, pp. 923-927.

[3] K. I. Diamantaras and M. G. Strintzis, "Camera motion parameter recovery under perspective projection," in IEEE International Conference on Image Processing, Sept. 16-19 1996, pp. 807-810.

[4] T. Papadimitriou, K. I. Diamantaras, M. G. Strintzis, and M. Roumeliotis, "Robust estimation of rigid-body 3-D motion parameters based on point correspondences," IEEE Transactions on Circuits and Systems for Video Technology, vol. 10, no. 4, pp. 541-549, June 2000.

[5] A. Chiuso, P. Favaro, H. Jin, and S. Soatto, "Structure from motion causally integrated over time," IEEE Transactions on Pattern Analysis and Machine Intelligence, vol. 24, no. 4, pp. 523-535, Apr. 2002.

[6] S. Soatto, R. Frezza, and P. Perona, "Motion estimation via dynamic vision," IEEE Transactions on Automatic Control, vol. 41, no. 3, pp. 393-413, Dec. 1996.

[7] M. Jankovic and B. K. Ghosh, "Visually guided ranging from observations of points, lines and curves via an identifier based nonlinear observer," Systems and Control Letters, vol. 25, pp. 63-73, 1995.

[8] X. Chen and H. Kano, "State observer for a class of nonlinear systems and its application to machine vision," IEEE Transactions on Automatic Control, vol. 49, no. 11, pp. 2085-2091, Nov. 2004.

[9] —_, "A new state observer for perspective systems," IEEE Transactions on Automatic Control, vol. 47, no. 4, pp. 658-663, Apr. 2002.

[10] W. E. Dixon, Y. Fang, D. M. Dawson, and T. J. Flynn, "Range identification for perspective vision systems," IEEE Transactions on Automatic Control, vol. 48, no. 12, pp. 2232-2238, 2003. 


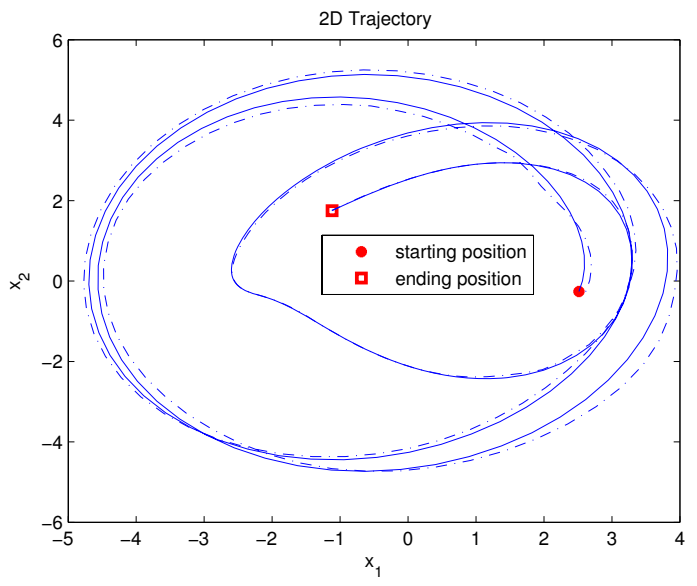

Fig. 8. 2D trajectory on the image plane of (18)

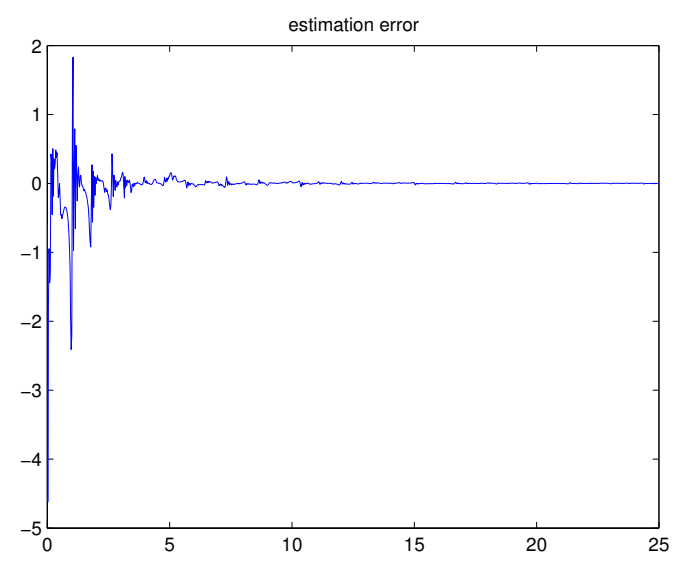

Fig. 9. State estimation error of $x_{3}$ of (19)

[11] D. Karagiannis and A. Astolfi, "A new solution to the problem of range identification in perspective vision systems," IEEE Transactions on Automatic Control, vol. 50, no. 12, pp. 2074-2077, Dec. 2005.

[12] L. Ma, Y. Chen, and K. L. Moore, "Range identification for perspective dynamic system with a single homogeneous observation," International Journal of Applied Mathematics and Computer Science, vol. 15, no. 1, pp. 63-72, 2005.

[13] O. Dahl, F. Nyberg, J. Holst, and A. Heyden, "Linear design of a nonlinear observer for perspective systems," in IEEE International Conference on Robotics and Automation, Barcelona, Spain, April 2005, pp. 429-435.

[14] L. Ma, Y. Chen, and K. L. Moore, "Perspective dynamic systems with 3D imaging surfaces," in American Control Conference, Portland, Oregon, USA, June 8-10 2005, pp. 3671-3675.

[15] S. Gupta, D. Aiken, G. Hu, and W. E. Dixon, "Lyapunov-based range and motion identification for a nonaffine perspective dynamic system," in Proceedings of the American Control Conference, Minneapolis, Minnesota, USA, June 14-16 2006, pp. 4471-4476.

[16] R. Y. Tsai and T. S. Huang, "Estimating three-dimensional motion parameters of a rigid planar patch," IEEE Transactions on Acoustic, Speech, and Signal Processing, vol. ASSP-29, no. 6, pp. 1147-1152, Dec. 1981.

[17] D. Shevitz and B. Paden, "Lyapunov stability theory of nonsmooth systems," IEEE Transactions on Automatic Control, vol. 39, no. 9, pp. 1910-1914, 1994.

[18] J. Pomet and L. Praly, "Adaptive nonlinear regulation: Estimation from the Lyapunov equation," IEEE Transactions on Automatic Control, vol. 37, no. 6, pp. 729-740, June 1992.
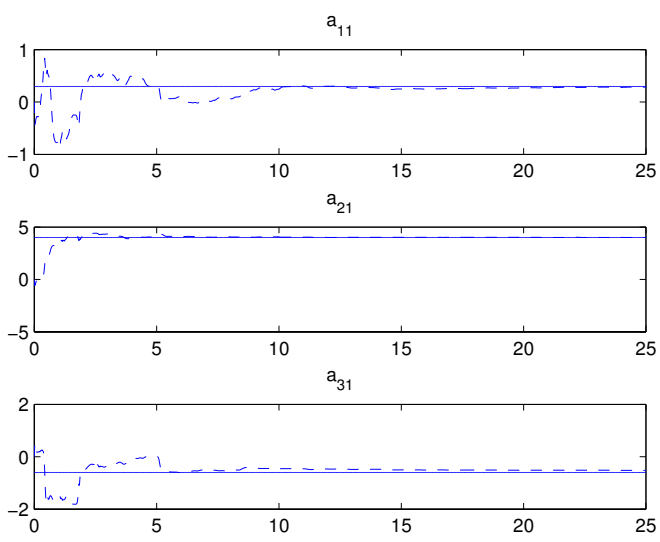

Fig. 10. Parameter estimation of $a_{i 1}$ (for $i=1,2,3$ ) of (19)
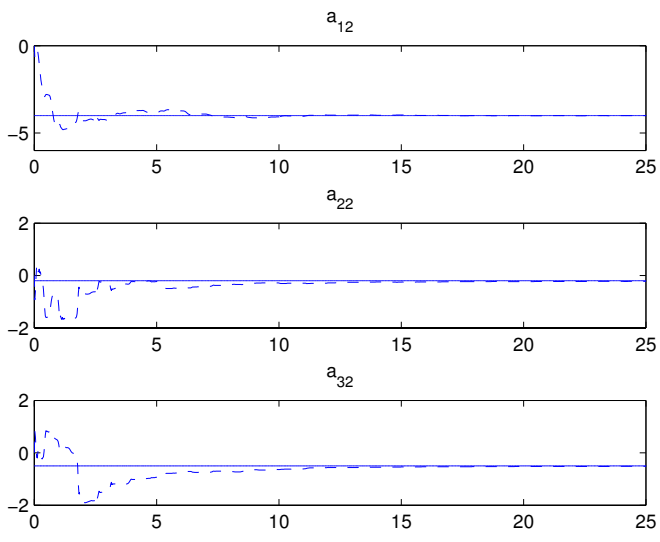

Fig. 11. Parameter estimation of $a_{i 2}$ (for $i=1,2,3$ ) of (19)
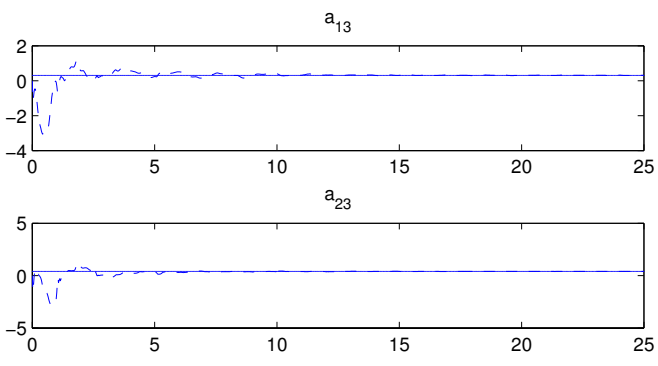

Fig. 12. Parameter estimation of $a_{13}$ and $a_{23}$ of (19) 